\title{
Morphology of corneal nerves and corneal sensation in dry eye: a preliminary study
}

\begin{abstract}
Purpose To evaluate the corneal sensitivity and nerve morphology in dry eyes.

Methods A total of 32 eyes of 16 patients (10

Sjogren's syndrome and six non-Sjogren's syndrome) and 19 eyes of 10 age-matched controls were studied. Sensitivity of the central cornea was measured by the Cochet-Bonnet aesthesiometer. The morphology of corneal nerves was studied by in vivo confocal microscopy (ConfoScan 2.0, Fortune Technologies Srl, Vigonza (PD), Italy). Sub-basal epithelial nerve plexus, subepithelial nerve plexus, and stromal nerves were localized and evaluated for the number of nerves, thickness, reflectivity, and tortuosity for each frame.

Results The mean corneal sensitivity of dry eye patients $(5.6 \mathrm{~mm} / \mathrm{grs} / \mathrm{S})$ was found significantly lower than that of the control $(5.0 \mathrm{~mm} / \mathrm{grs} / \mathrm{S})$ group $(P<0.01)$. Although the subepithelial nerves were thicker in dry eyes (mean: $6.38 \pm 1.18 \mu \mathrm{m}$ ) than control eyes (mean: $5.72 \pm 1.27 \mu \mathrm{m})$, this difference was not significant $(P>0.05)$.

Conclusion Decreased corneal sensitivity is not associated with morphological changes of corneal nerves in dry eyes.

Eye (2005) 19, 1276-1279. doi:10.1038/sj.eye.6701760; published online 19 November 2004
\end{abstract}

'Department of

Ophthalmology, Faculty of Medicine, Ankara University, Mamak, Ankara, Turkey

${ }^{2}$ Department of Biostatistics, Ankara University, Ankara, Turkey

Correspondence: N Örnek, Burcu sitesi no:16/31 451.sok, Çukurambar, Ankara 06520, Turkey Tel: + 90312 2840801; Fax: + 903123629782 E-mail: nurgulornek@ hotmail.com

Received: 13 June 2004 Accepted: 2 September 2004

Published online: 19

November 2004
Keywords: confocal microscopy; dry eye; corneal nerves; corneal sensitivity

\section{Introduction}

Dry eye is one of the most common eye disorders. ${ }^{1,2}$ Tear deficiency or excessive evaporation of the tears may cause this condition. ${ }^{1-3}$ Tear secretion is mainly caused by
BM Hoşal', N Örnek', G Zilelioğlu' and AH Elhan²

a reflex mechanism. Stimulation of the trigeminal nerve receptors in the cornea produce tear secretion from the lacrimal gland. ${ }^{3,4}$ Decreased corneal sensation reduces reflex tear secretion.

It is demonstrated that corneal sensitivity may decrease in dry eye patients. ${ }^{5}$ However, whether corneal hypoaesthesia is associated with morphological changes of corneal nerves in dry eye patients is not known.

In vivo confocal microscopy is a noninvasive technique that can image and measure the sublayers of the cornea at microscopic resolution. ${ }^{6,7}$ It is also used to image the corneal nerves successfully. ${ }^{4}$

The aim of this study is to evaluate and compare the corneal sensitivity and corneal nerve morphology in normal and dry eyes. As far as we are aware, corneal nerve morphology has not been previously studied in dry eye patients.

\section{Materials and methods}

\section{Patient selection}

In all, 32 eyes of 16 patients (15 female/one male) with dry eye (10 Sjogren's syndrome and six non-Sjogren's syndrome) and 19 eyes of 10 control subjects (eight female/two male) were studied.

Dry eye was defined as repetitive measurements of Schirmer I test (without anaesthetic) less than or equal to $5 \mathrm{~mm}$ at $5 \mathrm{~min}$, tear break-up time less than $10 \mathrm{~s}$, and associated corneal fluorescein staining.

None of the patients and controls were on systemic drugs that influence tear production. Dry eye patients with systemic or ocular disease related to corneal hypoaesthesia, who had previous contact lens wear or ocular surgery were excluded. Control patients did not have any ocular irritation, anterior segment disease, 
previous ocular surgery, contact lens wear, or systemic disease related to dry eye or corneal hypoaesthesia. They had a Schirmer test more than $15 \mathrm{~mm}$ at $5 \mathrm{~min}$. They were chosen from the employees of the eye department and patients presenting for routine eye examination.

\section{Measurement of corneal sensitivity}

Corneal sensitivity of the central cornea was measured by the Cochet-Bonnet aesthesiometer. The examination was initiated by a $60 \mathrm{~mm}$ length of nylon filament and continued by shortening it by $5 \mathrm{~mm}$ until the patient felt the contact of the filament. This length was then converted into pressure using a conversion table. The filament is applied perpendicularly to the central cornea. ${ }^{5}$ Corneal sensitivity was measured before the Schirmer and dye tests.

\section{Confocal microscopy unit}

The morphology of corneal nerves was studied by in vivo confocal microscopy (ConfoScan 2.0, Fortune Technologies Srl, Vigonza (PD), Italy). All the images were obtained with a standard $40 / 0.75 \mathrm{~W}$ objective lens, which has a field of approximately $340 \times 255 \mu \mathrm{m}^{2}$ (micrometers). The lateral resolutions of the images were approximately $1 \mu \mathrm{m}$, and the depth of resolution is approximately $10 \mu \mathrm{m}$.

\section{Confocal microscopic examination}

The patients were instructed about the procedure before the examination. A local anaesthetic solution was used before the examination in order to avoid blinking. After a drop of viscotears, gel was applied to the tip of the lens, the lens was advanced towards the central cornea until the gel contacted the cornea. The cornea was not touched by the objective lens. By moving the objective lens backward and forward, several layers of the cornea were imaged. The examination was carried out within $2 \mathrm{~min}$ on each eye. All the eyes were imaged twice. Nidek Advanced Vision Information System (NAVIS) was used to analyse the data. As a result of the diurnal variation of the corneal thickness, the imaging was performed between 1400 and 1700 hours.

\section{Classification of images}

The best focused images with the maximum nerve fibres were selected for examination. In our study, three layers of cornea were studied in order to evaluate the corneal nerves. ${ }^{4}$ The sub-basal epithelial cell layer is defined as the layer imaged just before Bowman's layer. It contains the sub-basal epithelial nerve plexus. The anterior stroma is defined as the layer just posterior to Bowman's layer. It can be imaged by confocal microscopy as the first frame after Bowman's layer. This layer contains subepithelial nerve plexus. The anterior and mid-stroma are the second and consecutive frames imaged by confocal microscopy and contain the stromal nerves. ${ }^{4}$

\section{Analysis of images}

The sub-basal epithelial nerve plexus, the subepithelial nerve plexus, and stromal nerves were localized and evaluated for the number of nerves, thickness (width), reflectivity, and tortuosity for each frame.

The tortuosity and the reflectivity of the nerve fibres were graded as described elsewhere. ${ }^{4}$ Briefly, tortuosity is graded from Grade 0 (the nerve fibres are almost straight) to Grade 4 (the nerve fibres are very tortuous), and reflectivity (brightness) is graded from Grade 0 (the nerve fibres are indistinguishable from the background) to Grade 4 (the reflectivity is much higher than the background).

\section{Statistical analysis}

Gender was evaluated by Fisher's exact test. Other variables were analysed by Mann-Whitney U test. A probability value $<0.05$ was considered to be statistically significant.

\section{Results}

The mean age of the patients with dry eye was 51 years (range, 30-69 years) and that of the healthy controls was 45 years (range, $34-65$ years) $(P>0.05)$. The mean corneal sensitivity of dry eye patients $(5.6 \mathrm{~mm} / \mathrm{grs} / \mathrm{S})$ was found significantly lower than that of the control $(5.0 \mathrm{~mm} / \mathrm{grs} /$ S) group $(P<0.01)$.

We could not demonstrate any morphological differences of corneal nerves between the groups. Although the subepithelial nerves were thicker in dry eyes (mean: $6.38 \pm 1.18 \mu \mathrm{m}$ ) (Figure 1) than control eyes (mean: $5.72 \pm 1.27 \mu \mathrm{m}$ ) (Figure 2) this difference was not statistically significant $(P>0.05)$. Table 1 demonstrates the characteristics of central corneal nerves.

\section{Discussion}

Lacrimal hyposecretion may develop due to a variety of congenital and systemic diseases, localized lacrimal disorders, Sjogren's syndrome (SS), and decreased corneal sensation. ${ }^{8}$ Dry eye is a very common disorder in the elderly population. ${ }^{2,9}$ Subclinical dacryoadenitis or senile atrophy and fibrosis of the human lacrimal gland may cause keratoconjunctivitis sicca in these patients. ${ }^{10}$ 
Corneal sensitivity diminishes with increasing age. ${ }^{11}$ This may also decrease tear secretion in the elderly population.

In ocular tissues, it is often difficult or impossible to obtain biopsy materials for ex vivo studies. ${ }^{6}$ Recently, the development of confocal microscopy allowed to image and measure sublayer thickness and cellular layer population of the cornea in situ at microscopic resolution. ${ }^{7}$ Confocal microscopy is used in the diagnosis of inflammatory and infectious keratitis, corneal dystrophies, epithelial downgrowth, and iridocorneal endothelial syndrome. It is also used to investigate the wound healing characteristics after photorefractive keratectomy, to measure flap thickness after laser-

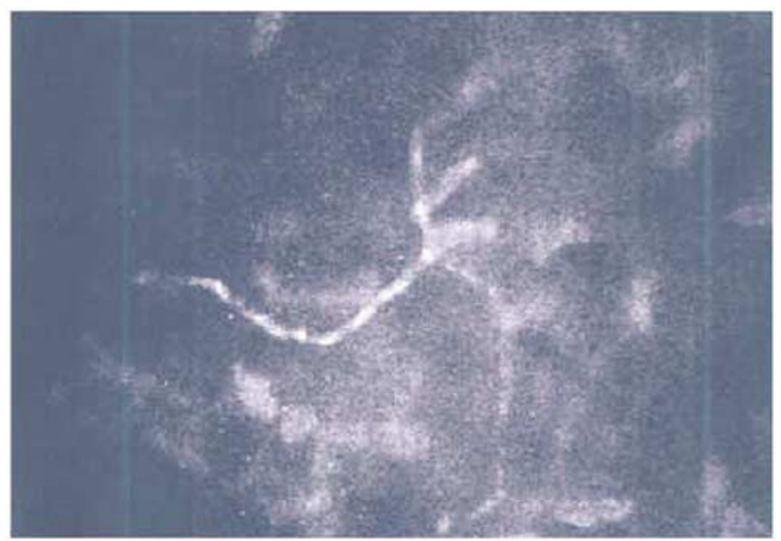

Figure 1 Corneal subepithelial nerve plexus in dry eye (nerve thickness, $7.9 \mu \mathrm{m}$ ). assisted in situ keratomileusis, and to examine the morphology of the tear film. ${ }^{6}$

Histological studies demonstrated that corneal nerves enter the cornea at the level of mid-stroma and run anteriorly and towards the central cornea. These nerves give rise to plexiform arrangements of progressively thinner nerve fibres at several levels within the cornea. After they form a dense subepithelial plexus, the nerves penetrate Bowman's layer and supply terminal endings to the corneal epithelium. ${ }^{12,13}$ Ultrastructural studies demonstrated that epithelial cells and keratocytes are directly innervated. ${ }^{14}$

Oliveira-Soto and associates evaluated the distribution and morphology of corneal nerves by confocal

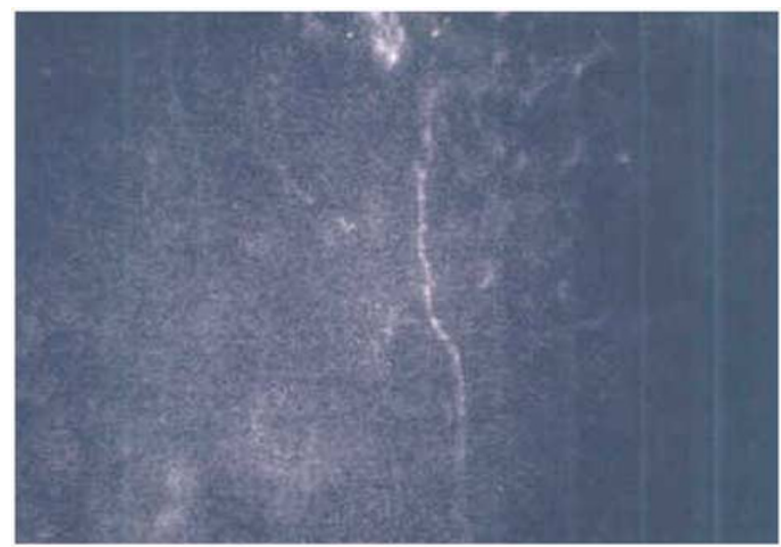

Figure 2 Corneal subepithelial nerve plexus in control eye (nerve thickness, $4.3 \mu \mathrm{m}$ ).

Table 1 Demonstration of the characteristics of central corneal nerves

\begin{tabular}{|c|c|c|c|c|c|}
\hline & \multicolumn{2}{|c|}{ Dry eyes } & \multicolumn{2}{|c|}{ Control eyes } & \multirow[t]{2}{*}{ P-value } \\
\hline & Mean $\pm S D$ & Median (min-max) & Mean $\pm S D$ & Median (min-max) & \\
\hline \multicolumn{6}{|c|}{ Sub-basal epithelial nerve plexus } \\
\hline Number of nerves & $2.03 \pm 2.10$ & $2(0-8)$ & $2.32 \pm 1.25$ & $2(0-5)$ & 0.23 \\
\hline Thickness $(\mu \mathrm{m})$ & $4.62 \pm 0.91$ & $4.55(3.3-6.3)$ & $4.68 \pm 0.68$ & $4.45(3.3-5.8)$ & 0.68 \\
\hline Tortuosity & $1.95 \pm 0.79$ & $2(1-3)$ & $1.83 \pm 0.62$ & $2(1-3)$ & 0.68 \\
\hline Reflectivity & $2.18 \pm 0.85$ & $2(0-3)$ & $2.33 \pm 0.49$ & $2(2-3)$ & 0.82 \\
\hline \multicolumn{6}{|l|}{ Subepithelial nerve plexus } \\
\hline Number of nerves & $1.31 \pm 1.60$ & $1(0-7)$ & $1.53 \pm 1.31$ & $1(0-6)$ & 0.25 \\
\hline Thickness $(\mu \mathrm{m})$ & $6.38 \pm 1.18$ & $6.40(3.9-8.7)$ & $5.72 \pm 1.27$ & $5.50(4.3-8.5)$ & 0.07 \\
\hline Tortuosity & $1.40 \pm 1.23$ & $1(0-4)$ & $1.29 \pm 0.99$ & $1(0-3)$ & 0.99 \\
\hline Reflectivity & $2.25 \pm 0.72$ & $2(1-4)$ & $2.24 \pm 0.44$ & $2(2-3)$ & 1.00 \\
\hline \multicolumn{6}{|l|}{ Stromal nerves } \\
\hline Number of nerves & $0.59 \pm 0.56$ & $1(0-2)$ & $0.63 \pm 0.50$ & $1(0-1)$ & 0 \\
\hline Thickness $(\mu \mathrm{m})$ & $10.12 \pm 2.62$ & $9.85(3.7-14.9)$ & $9.63 \pm 1.88$ & $9.4(7-12.9)$ & 0 \\
\hline Tortuosity & $0.22 \pm 0.94$ & $0(0-4)$ & $0.25 \pm 0.45$ & $0(0-1)$ & 0 \\
\hline Reflectivity & $2.61 \pm 0.61$ & $3(2-4)$ & $2.58 \pm 0.51$ & $3(2-3)$ & 0 \\
\hline
\end{tabular}

SD: standard deviation. 
microscopy. The authors demonstrated three corneal layers that contain nerve plexuses. The sub-basal epithelial cell layer, the layer between basal epithelial cells, and Bowman's layer, contain the sub-basal epithelial nerve plexus. These nerves are thought to be responsible for the innervation of the epithelium. The anterior stroma, the layer just posterior to Bowman's layer, contain the subepithelial nerve plexus. The anterior-mid and mid-stroma contain the stromal nerves. ${ }^{4}$ As a result of the limited capabilities of the technique, the authors could not comment on the nerves and the axon terminals that originated from the sub-basal epithelial nerves in the more superficial epithelial cell layers as described in histological examinations. Corneal nerves were not imaged in the posterior stroma,

Descemet's layer, and endothelial cell layer. ${ }^{4}$ The authors concluded that confocal microscopy is a suitable method to image corneal nerves. Their results were in accordance with the results obtained in previous histological studies. The limitations of this technique are that a small area of cornea can be examined at a time and the resolution of confocal microscopy is poor compared with the resolution of light and electron microscopy.

Corneal hypoaesthesia due to damage of the corneal nerves has been reported to decrease tear production after laser in situ keratomileusis. ${ }^{12}$ Decreased corneal sensitivity was also observed after contact lens wear. The authors suggested that the reduction in corneal sensitivity is due to sensory adaptation of cornea to the mechanical stimulation of the contact lens. ${ }^{15}$ Reduced corneal sensitivity improved after the cessation of contact lenses. ${ }^{11}$

Xe-Ping $\mathrm{Xu}$ and associates demonstrated decreased corneal sensitivity in dry eye patients. ${ }^{5}$ They thought that decreased corneal sensitivity is related to disturbance of the ocular surface integrity. In this study, increased staining was correlated with decreased corneal sensitivity. ${ }^{5}$ Sensory nerves may adapt to a irritation by decreasing the frequency and intensity of action potentials. Prolonged irritation of the cornea may elevate the pain threshold, and stronger stimuli is needed to evoke corneal sensation. ${ }^{5}$ In our study, the mean corneal sensitivity of dry eye patients was found significantly lower than that of the control group. Corneal hypoaesthesia may play a role in the pathogenesis of tear deficiency in these patients.

In this study, we studied the morphology of corneal nerves using confocal microscopy in dry eye patients and compared the results with normal age and sex-matched controls. Our findings could not demonstrate any significant difference in the morphology of the corneal nerves between the dry eyes and the control eyes. As a result of the limitations of this technique, we could not image the nerves in the superficial epithelial layers. In conclusion, although corneal sensitivity decreases in dry eye patients, it is not associated with morphological changes of corneal nerves. Sensory adaptation of cornea to the disrupted tear dynamics may cause corneal hypoaesthesia.

\section{References}

1 Liu Z, Pflugfelder SC. Corneal thickness is reduced in dry eye. Cornea 1999; 18(4): 403-407.

2 Lemp MA. New strategies in the treatment of dry-eye states. Cornea 1999; 18: 625-632.

3 Pflugfelder SC, Solomon A, Stern ME. The diagnosis and management of dry eye. A twenty-five-year review. Cornea 2000; 19: 644-649.

4 Oliveira-Soto L, Efron N. Morphology of corneal nerves using confocal microscopy. Cornea 2001; 20(4): 374-384.

$5 \mathrm{Xu}$ XP, Yagi Y, Tsubota K. Decrease in corneal sensitivity and change in tear function in dry eye. Cornea 1996; 15(3): 235-239.

6 Cavanagh HD, El-Agha MS, Petroll WM, Jester JV. Specular Microscopy, confocal microscopy and ultrasound biomicroscopy. Diagnostic tools of the past quarter century. Cornea 2000; 19(5): 712-722.

7 Cavanagh HD, Petroll WM, Jester JV. The application of confocal microscopy to the study of living systems. Neurosci Biobehav Rev 1993; 17: 483-498.

8 Kaufman HE, Barron BA, McDonald MB, Kaufman SC. Abnormalities of the tears and treatment of dry eyes. In: Kaufman HE et al (eds). Companion Handbook to the Cornea. Boston, Butterworth Heinemann: 2000, pp 29-42.

9 Strickland RW, Tesar JT, Berne BH, Hobbs BR, Lewis DM, Welton RC. The frequency of sicca syndrome in an elderly female population. J Rheumatol 1987; 14: 766-771.

10 Damato BE, Allan D, Murray SB, Lee WR. Senile atrophy of the human lacrimal gland: the contribution of chronic inflammatory disease. $\mathrm{Br}$ J Ophthalmol 1984; 68: 674-680.

11 Millodot M. Effect of long-term wear of hard contact lenses on corneal sensitivity. Arch Ophthalmol 1978; 96: 1225-1227.

12 Battat L, Marci A, Dursun D, Pflugfelder SC. Effects of laser in situ keratomileusis on tear production, clearance and the ocular surface. Ophthalmology 2001; 108: 1230-1235.

13 Müller LJ, Vrensen GFJM, Pels L, Cardozo BN, Willekens B. Architecture of human corneal nerves. Invest Ophthalmol Vis Sci 1997; 38: 985-994.

14 Müller LJ, Pels L, Vrensen GFJM. Ultrastructural organization of human corneal nerves. Invest Ophthalmol Vis Sci 1996; 37: 476-488.

15 Polse KA. Etiology of corneal sensitivity changes accompanying contact lens wear. Invest Ophthalmol Vis Sci 1978; 17: 1202-1206. 